\title{
Sparse coding and dictionary learning for spike trains to find spatio-temporal patterns
}

\author{
Taro Tezuka \\ From 24th Annual Computational Neuroscience Meeting: CNS*2015 \\ Prague, Czech Republic. 18-23 July 2015
}

In biological neural networks, it is widely accepted that the spikes are the fundamental building blocks of information representation [1]. In contrast, whether such building blocks exist at a higher level in terms of time and in a population of neurons is a topic of ongoing debate. One approach for finding candidates for such building blocks is to seek for frequently appearing spike patterns in a population. These sequences are often called spatio-temporal patterns, cell assemblies, or unitary events [2-4]. They could metaphorically be considered as an "alphabet" of neural information processing $[5,6]$. Some patterns have already been found and are related to functional roles such as memory consolidation and gating of sensory inputs $[7,8]$.

One difficulty in finding spatio-temporal patterns arises from observed spike trains being a superposition of multiple patterns. In signal processing, one commonly used method for decomposing the signal into patterns is dictionary learning for sparse coding [9-11]. Sparse coding expresses the input signal as a linear combination of a few template vectors taken from a matrix called a dictionary or codebook. In terms of linear algebra, sparse coding corresponds to finding a sparse vector $\mathrm{x}$, which fulfills $\mathrm{y}=\mathrm{Dx}$, where $y$ is the observed signal vector and $D$ is a dictionary. When the dimension of $x$ is much larger than that of $y$, it is possible to find sparse $\mathrm{x}$. Each column of $\mathrm{D}$ is called an atom, which represents a template vector. A good dictionary decomposes the most of the observed signals into a small set of template vectors. In other words, D must sparsify not just one input vector y but many others as well. This is represented by using matrix $Y$ whose column vectors are observed signals. In this case, sparse coding is represented by equation $\mathrm{Y}=\mathrm{DX}$. The goal is to find sparse matrix $\times$ given $\mathrm{Y}$ and $\mathrm{D}$. Whether input matrix $\mathrm{Y}$ can be transformed into sparse $\times$ or not depends on dictionary $\mathrm{D}$.

Correspondence: tezuka@slis.tsukuba.ac.jp

Faculty of Library, Information and Media Science, University of Tsukuba, Tsukuba, 305-0821, Japan 